\title{
ON THE OPTIMISATION OF A TEXTURE ANALYSER IN SQUEEZE FLOW GEOMETRY
}

\author{
$\underline{\text { Patrice Estellé }}^{1,4}$, Christophe Lanos ${ }^{2}$, Yannick Mélinge ${ }^{1}$, Colin Servais ${ }^{3}$ \\ ${ }^{1}$ LGCGM, Département Matériaux et Thermique de l'Habitat, Institut National des Sciences Appliquées de Rennes, \\ 20 avenue des Buttes de Coësmes, 35043 Rennes Cedex, France \\ ${ }^{2}$ LGCGM, Département Matériaux et Thermique de l'Habitat, Institut Universitaire Technologique, \\ rue du Clos Courtel , BP 90422, 35704 Rennes Cedex 7, France \\ ${ }^{3}$ Nestlé Research Center, Vers-chez-les-Blanc, 1000 Lausanne 26, Switzerland \\ ${ }^{4}$ Correspondence concerning this paper should be addressed to \\ Patrice Estellé \\ LGCGM - Laboratoire Matériaux et Thermique de l'Habitat \\ INSA Rennes \\ 20 avenue des Buttes de Coësmes \\ 35043 Rennes Cedex France \\ Tel: +33(0) 223238200 \\ Fax: +33 (0) 223238448 \\ E-mail: patrice.estelle@insa-rennes.fr
}

\begin{abstract}
This paper describes how the range of application of a texture analyser, used for mechanical tests of solids and liquids in the food and cosmetics industry, can be extended to reproduce squeeze flow geometry. It describes the necessary optimisation of the device to ensure parallelism and thermal regulation of the plates during tests. The error on the load cell and the instrument compliance are evaluated. The influence of these artefact measurements is investigated in terms of interpretation of rheological properties of materials.
\end{abstract}

Keywords: texture analysers, squeezing flow, calibration, instrument compliance, plate parallelism, thermal regulation, rheometry. 


\section{Introduction}

Texture analysers are commonly used to investigate the mechanical response directly linked to the surface or the internal structure of materials. This is performed with different appropriate probes which differ from the type of tested materials. In the food industry, these instruments are used in product development and in quality control [1]. Mechanical results are dependent of the type of probe used and are essentially comparative [2], and complete chemical and calorimetric analyses. The whole set of data is supposed to bring complementary information to sensorial analysis, which can be performed only by a trained panels of experts.

The aim of this paper is to extend the range of application of an existing texture analyser in order to perform unconfined compression between parallel plates or squeeze flow [3]. The interest of such adaptation is mainly to provide a potentially cheaper alternative to existing squeeze flow rheometers. So, texture analysers are widely used in food and cosmetic laboratories, compare to rigid presses which are frequently used in other industries processing stiffer materials. Furthermore, the squeeze flow is relatively easy to perform and it is used increasingly as a means for measuring the biaxial extensional viscosity [4], the liquid-solid relative motion of pastes [5,6] and the rheological properties of many fluids [7]. It appears that the squeeze flow geometry is also an interesting alternative to shear rheological techniques, particularly for concentrated suspensions [1] and for sensitive fluids. Actually, in this last case, the advantage of the squeeze flow geometry is that the fluid structure is not disturbed prior the measurement [8]. In addition, several analytical solutions of such a flow are proposed for different rheological behaviour and boundary conditions [4,7-13]. In principle, this enable the computation of material properties by fitting experimental data with analytical solutions $[7,10]$.

In this flow geometry, a fluid sample is squeezed between two circular coaxial parallel plates, without superimposed rotation. Such a flow is radial and axial, inherently transient and spatially inhomogeneous due to the changing geometry. The reference parameters of the flow are the radius 
$R$ of the plates or the sample, the height $h$ of the sample, the compression speed $\dot{h}$ and the compression load $F$. These parameters are imposed or recorded during the test.

The purpose of this note is to describe how the performance of a texture analyser can be improved in order to be used as a squeeze flow geometry. This involves a series of modifications of the device and its calibration. The details of modifications are presented in the following section. Artefact measurements are also evaluated and their influence on the interpretation of the squeeze flow raw data in terms of material rheological properties are finally discussed using theoretical arguments.

\section{Instrument description}

This study is based on the TA-XT2i@ texture analyser from Stable Micro Systems ${ }^{\circledR}$. A schematic side view of the device is shown in figure 1 . The load cell (strain gauge type) of the texture analyser is placed in the mobile arm of the device. Here, a $250 \mathrm{~N}$ load cell is used; its accuracy is $0.01 \mathrm{~N}$ (manufacturer data). The mobile arm moves in translation up or down the column. The arm displacement, limited and secured by two sensors, is ensured by a stepper drive motor. In our squeeze flow geometry, the squeezing action is realised by moving the upper plate at constant compression speed $\dot{h}$, with the lower one remaining motionless. Consequently, the recorded applied force varies with time. In order to ensure the symmetry of the squeeze flow geometry, the first modification concerns the original test fixture of the texture analyser which is modified with an additional base. It may also supports a plate similar to the highest one (see figure 1). Design is evolved to guarantee a simple and fast installation of plates of various diameters. Additional base and plates are made of steel.

Moreover, it is well known that plate parallelism and thermal homogeneity of the plate surface are necessary to ensure an axisymmetric radial flow. As shown in [13], a parallelism default causes flow in a preferential direction. In the same way, a temperature gradient at the plate surface would induce an asymmetry of the velocity field, which then complicates the squeeze flow analysis [14]. 
To overcome the problem of plate parallelism, the upper plate is screwed onto a mounting plate, itself fixed on the load cell (see figure 1). The upper plate rests on three ball screws used for adjustment of parallelism before a test. The three ball screws are spaced at $120^{\circ}$ intervals around the plate.

Secondly, a thermal regulation system is developed to control the plate temperature. In fact, the drive mechanism installation under the bottom plate heats up that plate to $25 \mathrm{deg} \mathrm{C}$ if the instrument is placed in a room at $18 \mathrm{deg} \mathrm{C}$, as shown in figure 2 . This fact has not been mentioned by previous authors which have used the same device to adapt an imperfect squeeze flow geometry and test food samples, see [15] as example. To avoid this problem, a temperature control system using a fluid flowing through a pipe network machined into both test plates is thus developed. The $5 \mathrm{~mm}$ diameter network pipes are connected to a thermostatic water bath using flexible pipes. To improve the temperature regulation efficiency, the temperature of each plate is recorded over time, as well as that of the instrument base and the room temperature during a 8 hours test. Figure 3 gives an example of such temperature regulation for a target plate temperature of $24 \mathrm{deg} \mathrm{C}$, above the temperature room and below the temperature drive. In this test, the water bath was started 2 hours after the texture analyser in order to evaluate the reaction time of the thermal regulation system and the initial temperature difference between both plates. Figure 3 shows that a stable temperature difference between the plates of $0.5 \operatorname{deg} \mathrm{C}$ was reached within a few minutes. This temperature difference is sufficient for the present purpose and vanishes when tests are carried out at temperatures that are higher than the drive temperature or when temperature regulation is close to the room temperature [16]. Consequently, the described modifications provide experimental conditions needed to squeeze flow measurements. To ensure the right measurement of key parameters of squeeze flow (the squeeze force $F$ and the height $h$ of the sample) the adapted device is calibrated as described below. 


\section{Calibrations}

\subsection{Force transducer}

As indicated by the manufacturer, calibration of the force transducer must be performed by placing, with the $250 \mathrm{~N}$ load cell, a $50 \mathrm{~N}$ weight on the calibration platform of the mobile arm. This calibration is carried out for each geometry test. The load cell is equipped with fixed strain gauges that enable the control of profile deformation. The technological choice of strain gauges here is satisfactory because of the low range of compression speeds. In order to calibrate the load cell, a repeated measured of applied loads $(0.05,0.1,1$ and $10 \mathrm{~N})$ is realised during loading and unloading steps. Load cell calibration allows the determination of calibration curve, as shown by equation (1).

$$
\mathrm{F}_{\text {real }}=0.9987 \times \mathrm{F}_{\text {read }}+1.345 \times 10^{-2}
$$

where $F_{\text {real }}(\mathrm{N})$ denotes the applied load and $F_{\text {read }}(\mathrm{N})$ is the measured load.

However, replicates carried out in the range $0.05 \rightarrow 10 \mathrm{~N}$ show that the error associated to this measurement can be only neglected above $1 \mathrm{~N}$ (figure 4). In practice, the user can set the data acquisition of the texture analyser for a minimum trigger force. This minimum trigger force corresponds to a contact detection between the upper plate and the tested material. According to the results obtained in figure 4 , the default value of the trigger force, which generally determines the plate position where the gap vanishes, must be revised and set to $1 \mathrm{~N}$. With a $1 \mathrm{~N}$ trigger force, the measurement error is lower than $1 \%$ and instrument compliance is low, as it will be shown before.

\subsection{Displacement}

The displacement of the mobile arm of the texture analyser is ensured by a stepper drive motor. A cycling measurement of a displacement issued from a reference point is carried out and checked with a comparator distance with an accuracy of $10^{-2} \mathrm{~mm}$. The type of plates (various weight) and the compression speed of the upper plate do not have any influence on the measure. Indeed, results agree well with displacement instruction, the relative standard deviation of the displacement being 
$10^{-2} \mathrm{~mm}$. Statistical study of the displacement signal [16] shows that the error linked to the compression speed is of the same order of magnitude. However, this study shows that acceleration and slowing down phases appear during the arm displacement, respectively at the start and the stop of its movement.

\subsection{Instrument compliance}

An accurate knowledge of sample height is essential to correctly interpret squeeze flow data [13]. Because of the device design (figure 1), compliance must be corrected over the accessible range of the load cell. For this purpose, one determines the total deflection of the instrument as a function of the applied load by letting the upper plate press directly on the lower plate until the overload value was reached and recorded the apparent plate displacement (figure 5). The compression speed and the plate set-up used are identical to those used for a real test. In this manner, one could estimate the real height of the sample during real tests. For a given compression load $F$, the real height $h_{\text {real }}$ $(\mathrm{mm})$ of a sample is obtained by the following relationship:

$$
\mathrm{h}_{\text {real }}=\mathrm{h}_{\text {measured }}-\mathrm{h}_{\text {compliance }}(\mathrm{F})
$$

where $h$ measured denotes the gap between the plates during a squeeze test and $h$ compliance $(F)$ is the instrument compliance corresponding to the applied load.

Figure 5 shows that, for significant load values, the instrument compliance is about to $1 \mathrm{~mm}$. The texture analyser software cannot automatically make the correction suggested by equation (2), but this can be performed a posteriori when data are numerically treated.

Due to the instrument compliance, the plate parallelism must be checked during test phase. The experimental operation developed to check parallelism is depicted in figure 6. Four load cells are spaced in a square pattern on a $50 \mathrm{~mm}$ radius plate. These load cells work mainly in compression and act like a coiled spring. We assumed that the stiffness of the load cells is constant and corresponds to a displacement of $0.013 \mathrm{~mm}$ for a $1000 \mathrm{~N}$ force (manufacturer data). Performance 
tests without any sample are carried out in five replicates using two compression speeds $(0.2$ and $\left.0.5 \mathrm{~mm} . \mathrm{s}^{-1}\right)$. Similar results are obtained for both compression speeds, consequently only the lowest one is discussed below. The initial height between the upper plate and the top of the four load cells is $5 \mathrm{~mm}$. Figure 7 shows that the force results from the texture analyser and from the four load cell system are in agreement. Moreover, the load distribution is not equivalent on each cell. A deviation is estimated from these values, which takes into account the flexion of the mobile arm. Figure 8 shows that the deviation from perfect parallelism appears to be extremely very low $\left(<3 \times 10^{-5} \mathrm{rad}\right)$, which proves that instrument compliance does not alter plate parallelism in measurement conditions.

\section{Discussion}

The quantification of the influence of artefact measurement described in this work, due to error on the load cell and instrument compliance, is analysed in terms of the interpretation of the squeeze flow raw data from a theoretical point of view. For this, we consider simulated sticking squeeze flow data of a Newtonian fluid. Such a solution is known [7] and can be expressed in the reduced system $\mathrm{F}^{*}(\mathrm{~h} / \mathrm{R})[6]$.

$$
F^{*}=\frac{3 \alpha \dot{h}}{2 R}\left(\frac{R}{h}\right)^{2}
$$

with $\mathrm{F}^{*}=-\frac{\mathrm{Fh}}{\pi \mathrm{R}^{3}}$ and $\alpha$ is the Newtonian viscosity (Pa.s).

For squeeze flow simulation, the calculation of the reduced squeeze force $F^{*}$ is obtained with a Newtonian viscosity $\alpha$ of 1 Pa.s and a ratio $\dot{h} / R$ equals to $4.10^{-2} \mathrm{~mm} . \mathrm{s}^{-1}$. The gap to radius ratio $h / R$ varies between 1 and $10^{-2}$, with an interval of $10^{-3}$ between each point. This theoretical solution is compared with the same solution including the artefact measurements. Results are shown in figure 9 . In this example, for $h / R=0.1$ and 0.7 , an error of $10 \%$, respectively $1.3 \%$, on the measurement of the sample height due to the instrument compliance, added to an error of $0.3 \%$, 
respectively $14 \%$, on the measurement of the compression load due to the error on the load cell will imply an error of $20 \%$, respectively $17 \%$, on the fitted viscosity according to the raw data interpretation published in $[7,10]$. Error on the viscosity is shown on figure 10 . With our corrections of artefact measurements, these errors are substantially reduced and material rheological properties can be accurately determined.

\section{Conclusion}

We have extended the range of application of a texture analyser to perform squeeze test. Simple modifications have been first developed to ensure plate parallelism during tests and to improve the temperature stability of the plates. A comprehensive calibration of the device was also realised. Results of the load cell calibration lead to a modification of the trigger force value initially used in the software in order to correctly determine the plate position. The principal reason for this modification is the uncertainty of the sample height. Another important consideration is the instrument compliance and its effect on the plate parallelism and the force distribution on the plate surface. We have shown that parallelism adjustment obtained from ball screws system is not modified by the instrument compliance. Our results have also shown the requirement of the instrument compliance measurement to determine accurately the distance between the plates or the sample height, to correctly evaluate the material properties in squeeze flow geometry.

\section{Acknowledgments}

The authors would like to thank G. Brigant from INSA Rennes for the design of the new different parts of the device and his support in the experiments.

\section{References}

[1] H. A. Barnes, A review of the slip (wall depletion) of polymer solutions, emulsions and particle suspensions in viscometers: Its cause, character and cure, Journal of Non-Newtonian Fluid Mechanics 56 (1995) 221-251. 
[2] M. C. Bourne, Texture profile analysis, Food Technology 32 (7) (1978) 62-66.

[3] J. R. Scott, Theory and application of the parallel plate plastimeter, Trans. Inst. Rubber Ind. 7 (1931)169-186.

[4] S. Nasseri, L. Bilston, B. Fasheun, R. Tanner, Modelling the biaxial elongational deformation of soft solids, Rheologica. Acta 43 (2004) 68-79.

[5] J. D. Sherwood, Liquid-solid relative motion during squeeze flow of pastes, Journal of NonNewtonian Fluid Mechanics 104 (2002) 1-32.

[6] N. Roussel, C. Lanos, Y. Mélinge, Induced heterogeneity in saturated flowing granular media Powder Technology 138 (2003) 68-72.

[7] G. H. Meeten, Squeeze flow of soft solids between rough surfaces, Rheologica. Acta 43 (2004) 6-16.

[8] D. N. Smyrnaios, J. A. Tsamopoulos, Squeeze flow of Bingham plastics Journal of NonNewtonian Fluid Mechanics 100 (2001) 165-190.

[9] M. J. Adams, I. Aydin, B. J. Briscoe, S. K. Sinha, A finite element analysis of the squeeze flow of an elasto-viscoplastic paste material, Journal of Non-Newtonian Fluid Mechanics 71 (1997) 4157.

[10] J. D. Sherwood, D. Durban, Squeeze flow of power law viscoplastic solid, Journal of NonNewtonian Fluid Mechanics 62 (1996) 35-54.

[11] N. Roussel, C. Lanos, Plastic fluid flow parameters identification using a simple squeeze test Applied Rheology 13 (3) (2003) 132-141.

[12] P. Estellé, C. Lanos, Y. Mélinge, C. Servais, Squeezing flow for rheological characterisation of food materials, in Proc 3rd Int. Symp. Food Rheology Structure, Zürich, 2003, pp.115-119.

[13] M. J. Adams, B. Edmonson, D. G. Caughey, R. Yahya, An experimental and theoretical study of the squeeze-film deformation and flow of elastoplastic fluids, Journal of Non-Newtonian Fluid Mechanics 51 (1994) 61-78.

[14] S. Hess, A. Sow, Squeeze-flow in the presence of a temperature gradient: effective attraction between asperites in the friction zone of two solids, Journal of Non-Equilibrium Thermodynamics 27 (2) (2002) 121-130.

[15] T. Suwonsichon, M. Peleg, Imperfect squeezing flow viscometry of mustard with suspended particulates, Journal of Food Engineering 39 (1999) 217-226.

[16] P. Estellé, Méthodes d'analyse inverse des données d'écoulement de compression de fluides complexes homogènes, Thesis Insa Rennes, 2004. 


\section{Figure caption}

Figure 1. Schematic side view of the TA-XT2i@ texture analyser from Stable Micro Systems ${ }^{\circledR}$ used for squeezing flow geometry adaptation. The original test fixture is modified with an additional base. The lower plate is fixed on this additional base with screws.

Figure 2. Temperature control of the plates over a 8 hour period.

Figure 3. Influence of the thermal regulation system on the plate temperature.

Figure 4. Load cell calibration: error on the recorded load value.

Figure 5. Instrument compliance measurement.

Figure 6. Schematic of the system developed to check the plate parallelism during tests - (a) Side view - (b) Top view of the system, $F_{i}$ denotes the load cell position.

Figure 7. Comparison of force values recorded by the texture analyser software and by the four load cells.

Figure 8. Deflection of plate parallelism against compression force.

Figure 9. Comparison of the theoretical reduced squeeze force of a Newtonian fluid in sticking flow with (thin line) and without (thick line) the influence of artefact measurements.

Figure 10. Viscosity of a Newtonian fluid in simulated sticking flow with (thin line) and without (thick line) the influence of artefact measurements. 


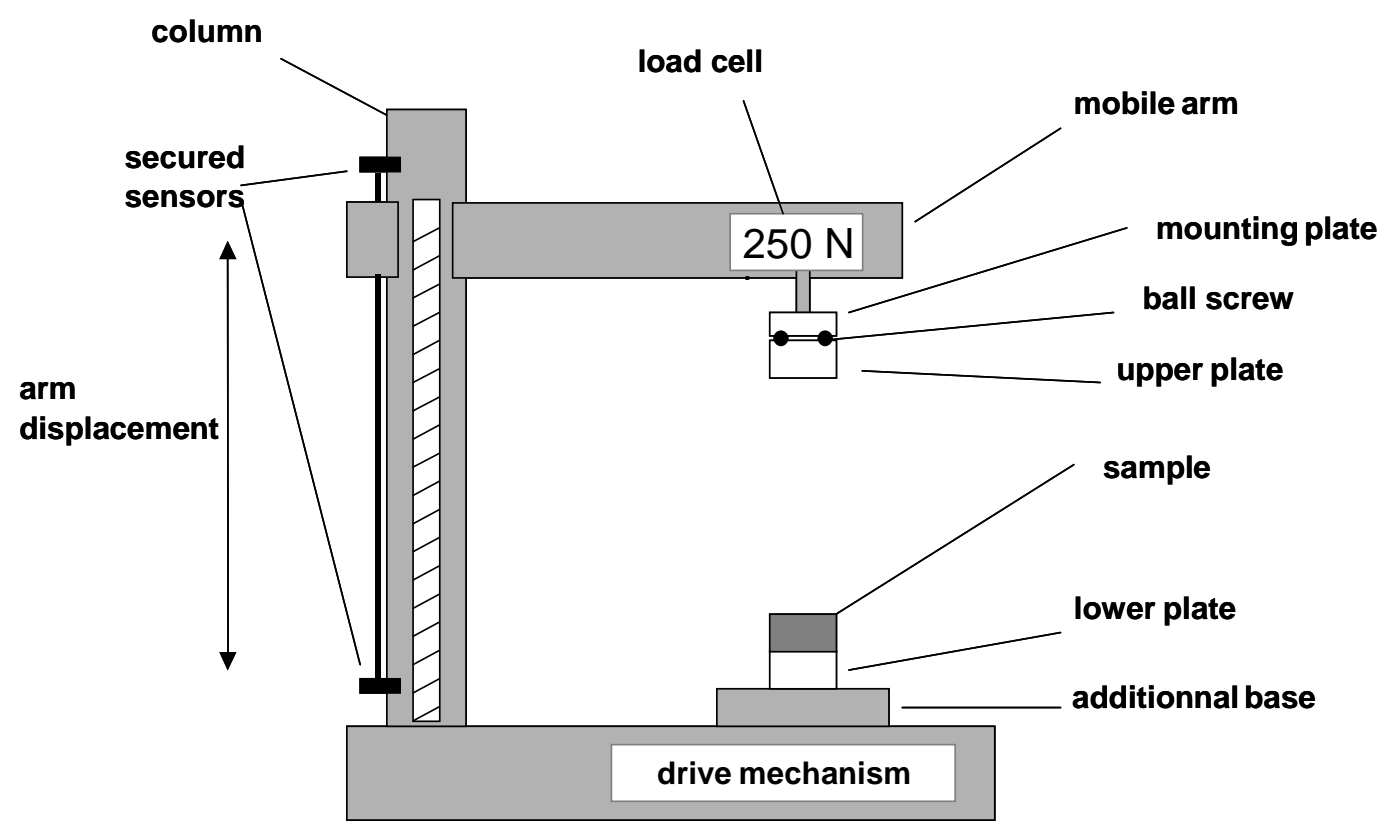

Figure 1. Schematic side view of the TA-XT2i®C texture analyser from Stable Micro Systems ${ }^{\circledR}$ used for squeezing flow geometry adaptation. The original test fixture is modified with an additional base. The lower plate is fixed on this additional base with screws. 


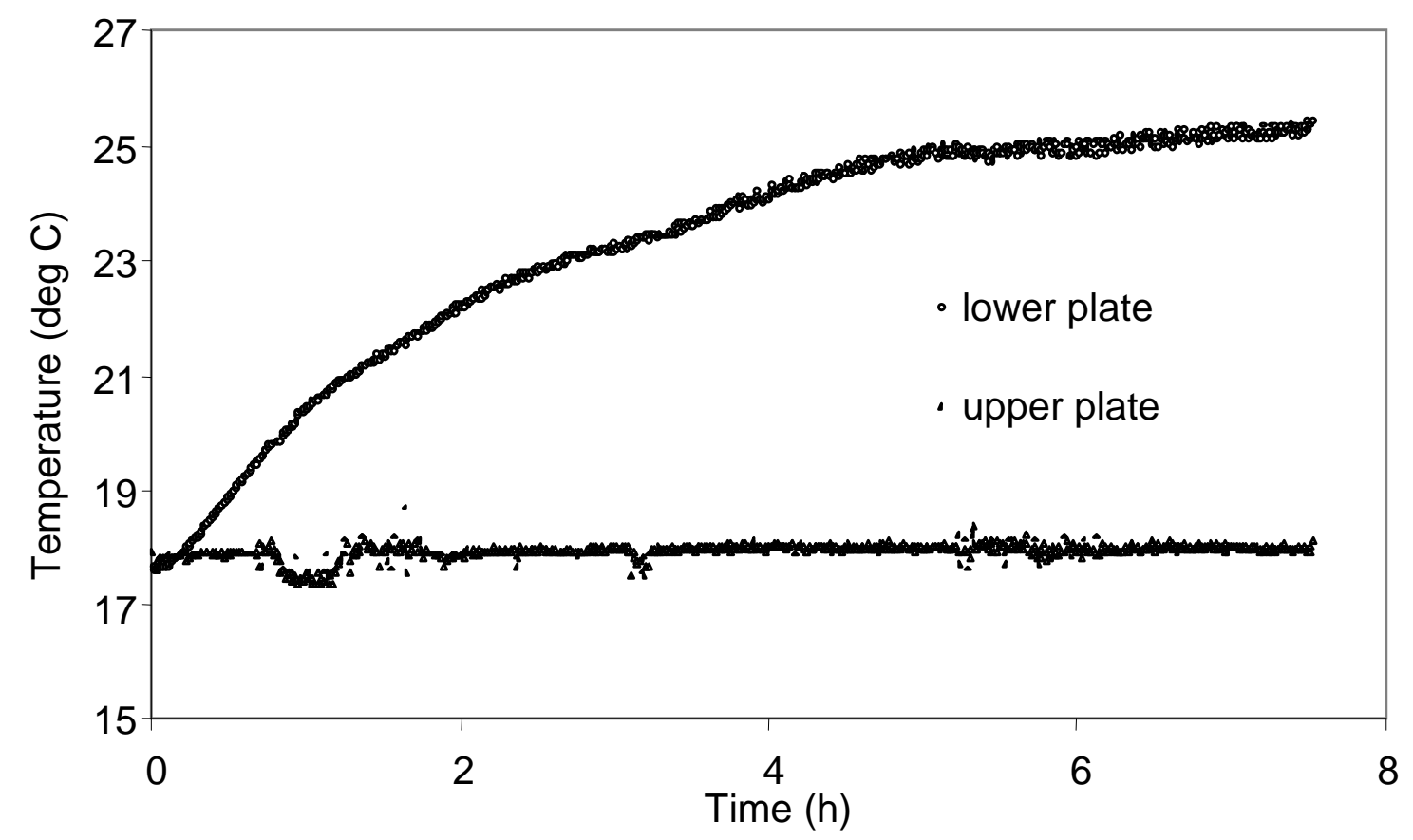

Figure 2. Temperature control of the plates over a 8 hour period. 


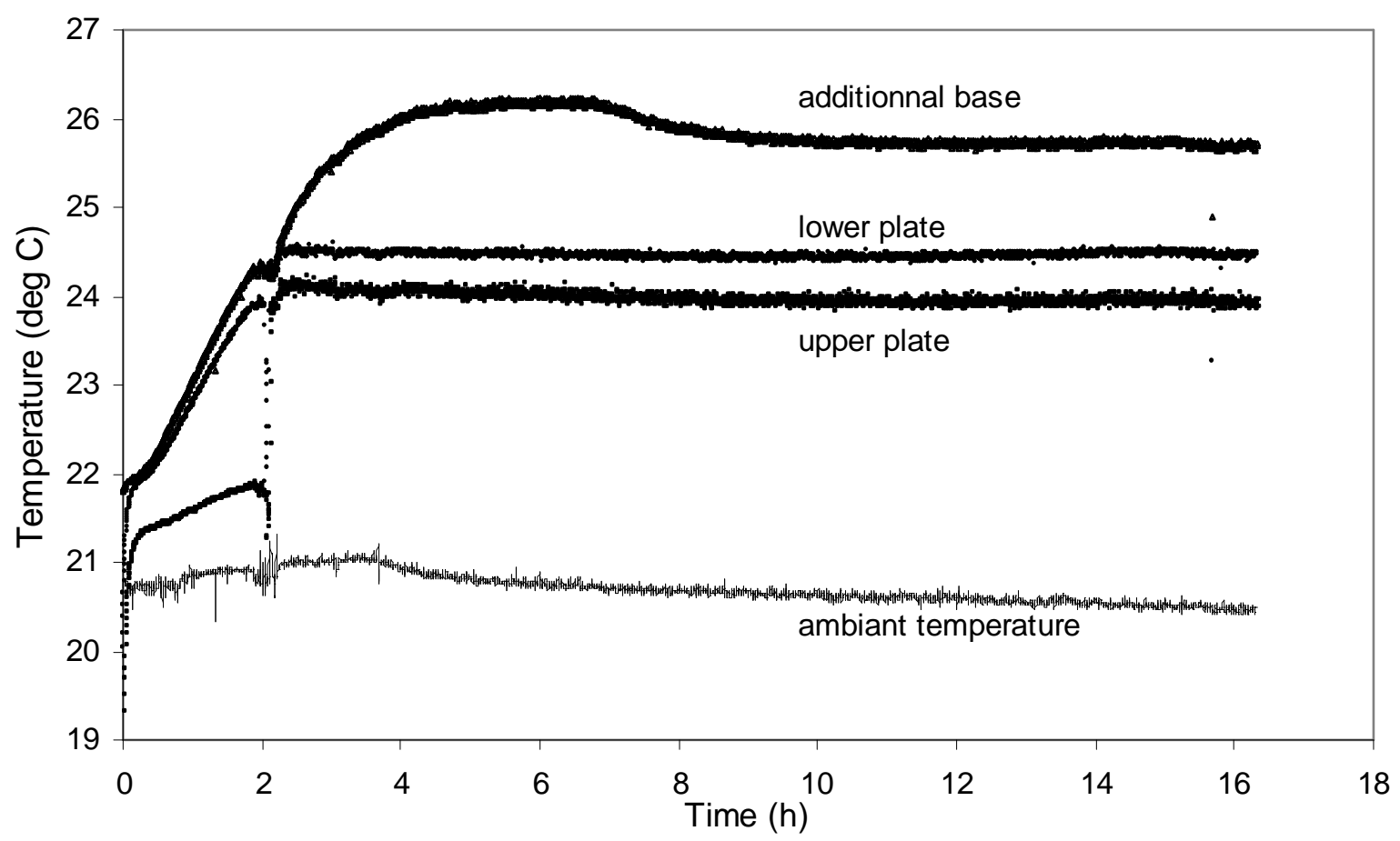

Figure 3. Influence of the thermal regulation system on the plate temperature. 
Estellé et al Measurement

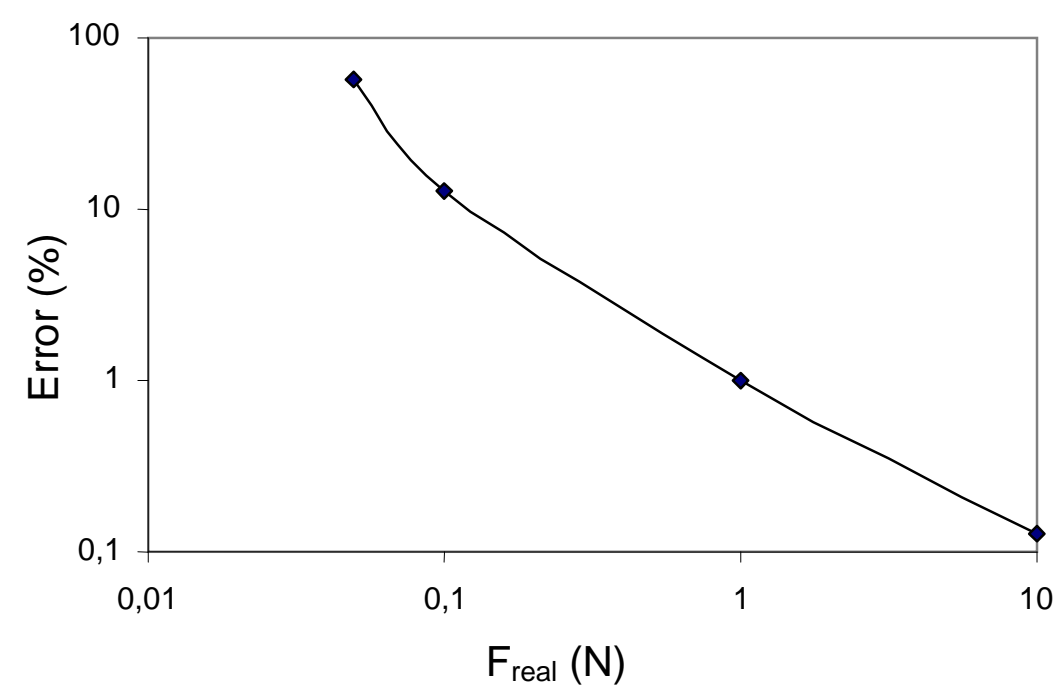

Figure 4. Load cell calibration: error on the recorded load value. 


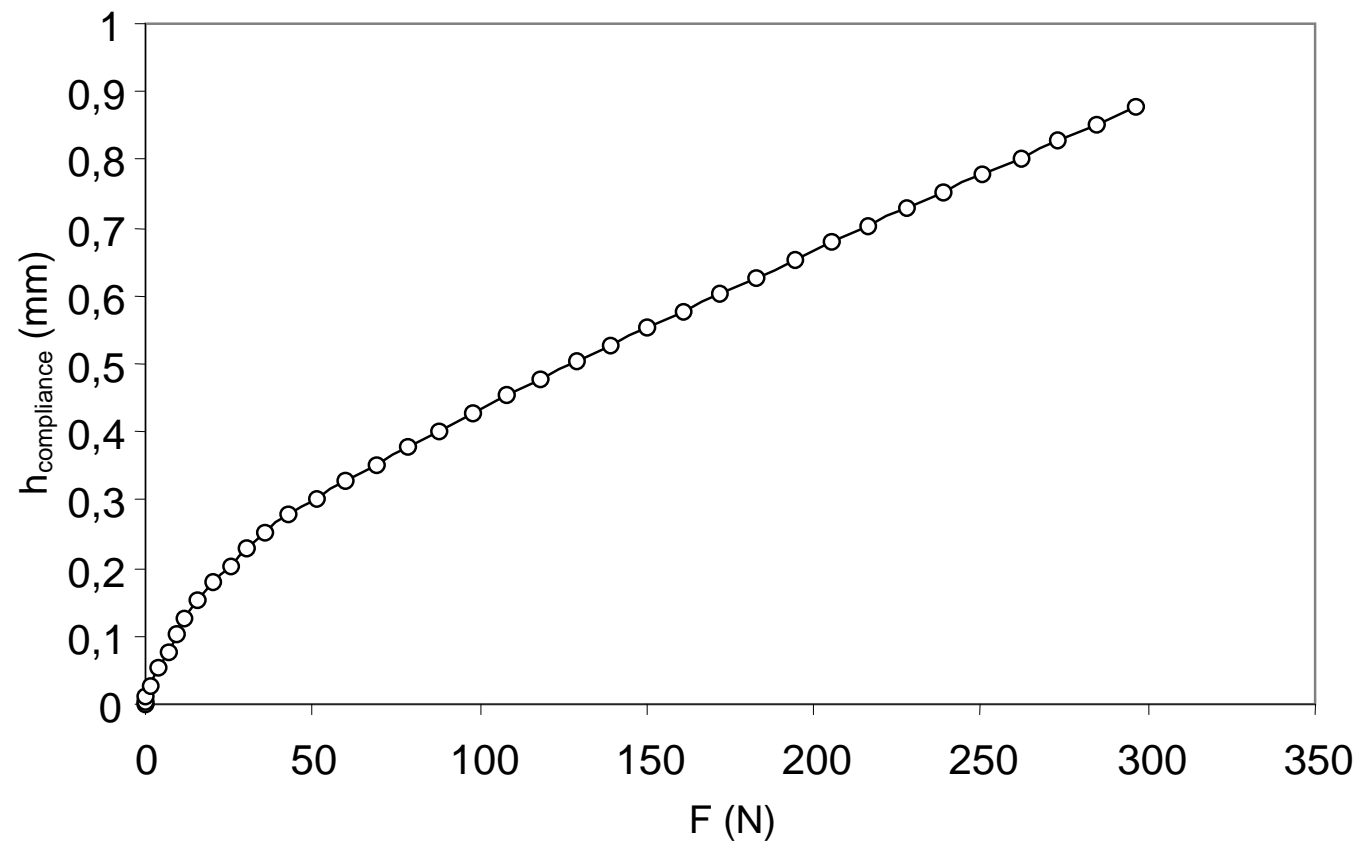

Figure 5. Instrument compliance measurement. 


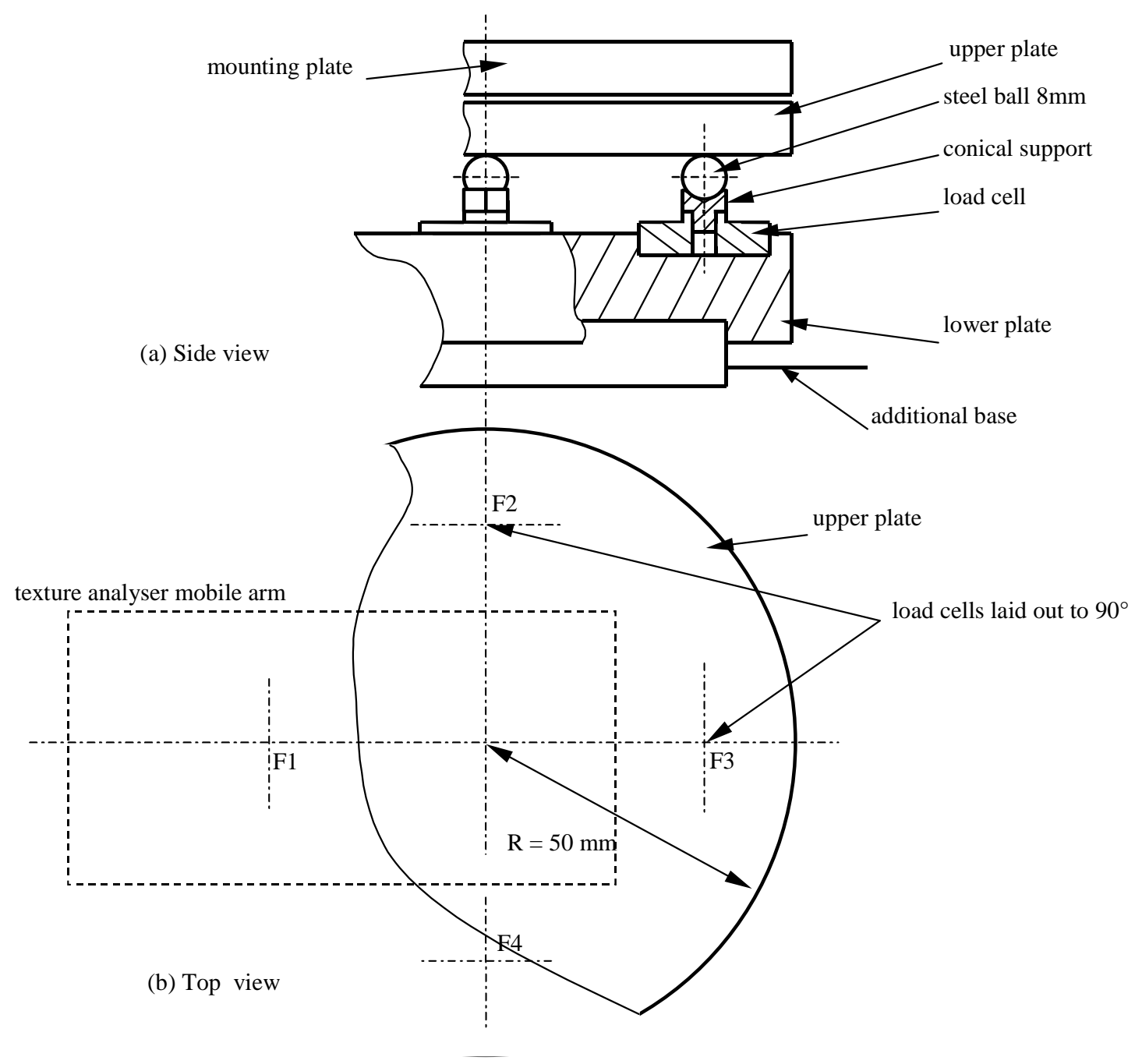

Figure 6. Schematic of the system developed to check the plate parallelism during tests - (a) Side view - (b) Top view of the system, Fi denotes the load cell position. 
Estellé et al Measurement

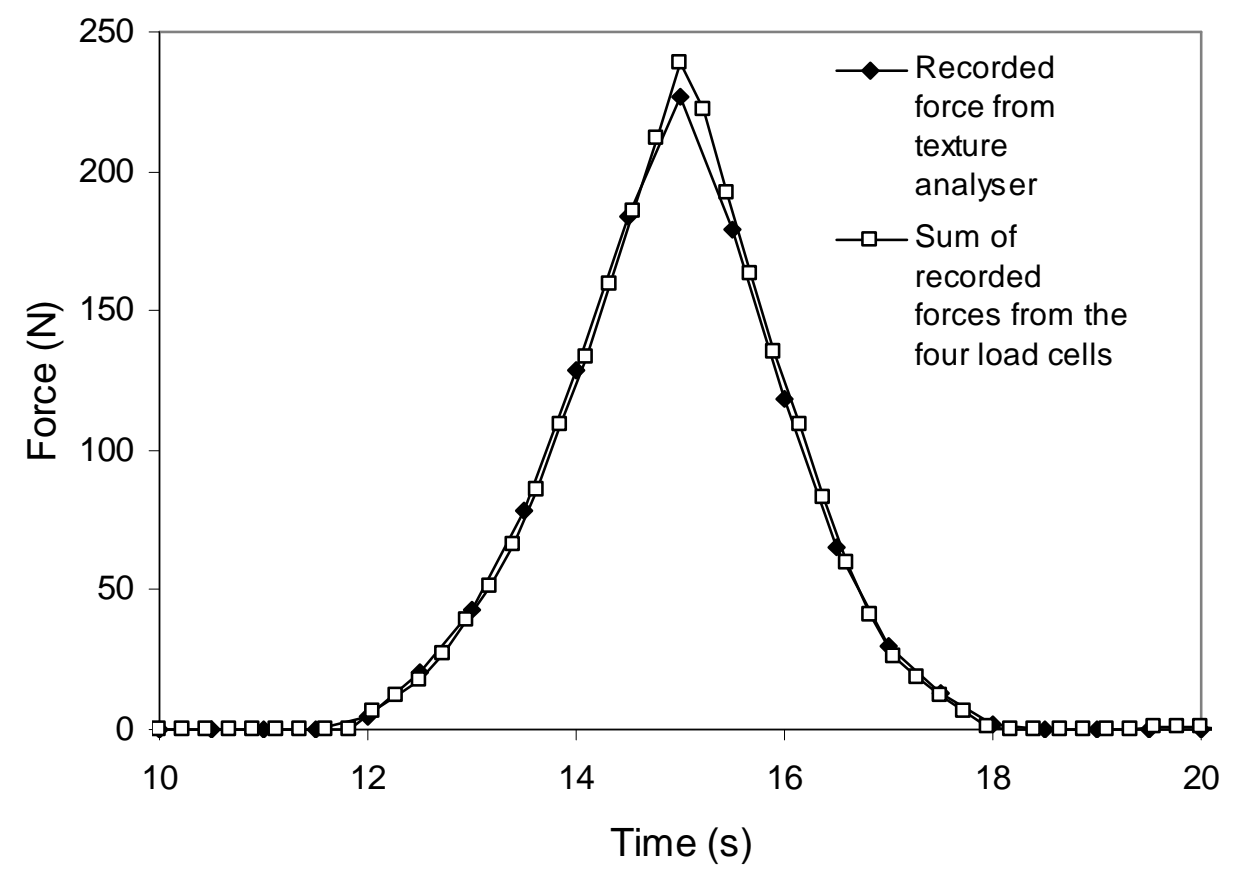

Figure 7. Comparison of force values recorded from texture analyser and from the four load cells. 


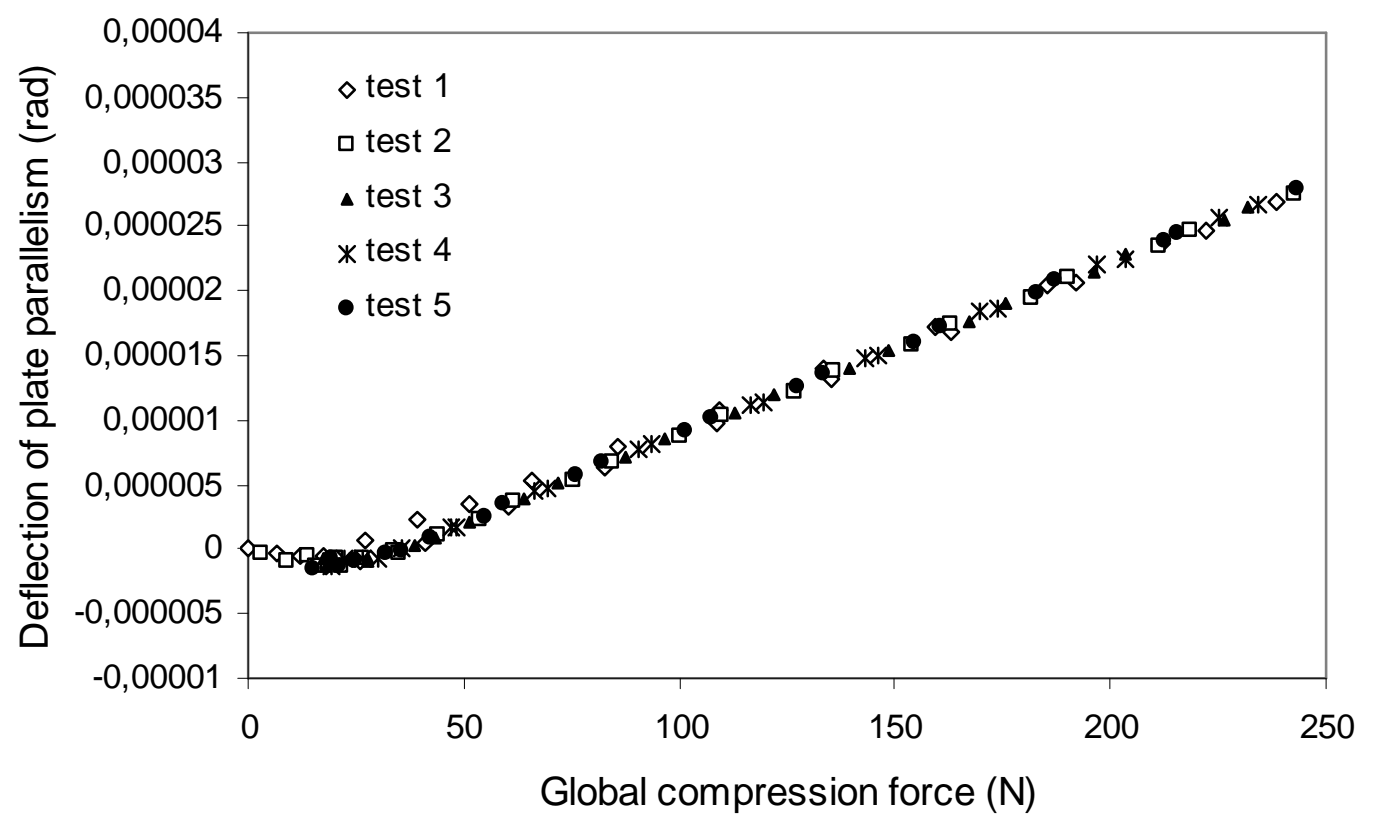

Figure 8. Deflection of plate parallelism against compression force. 


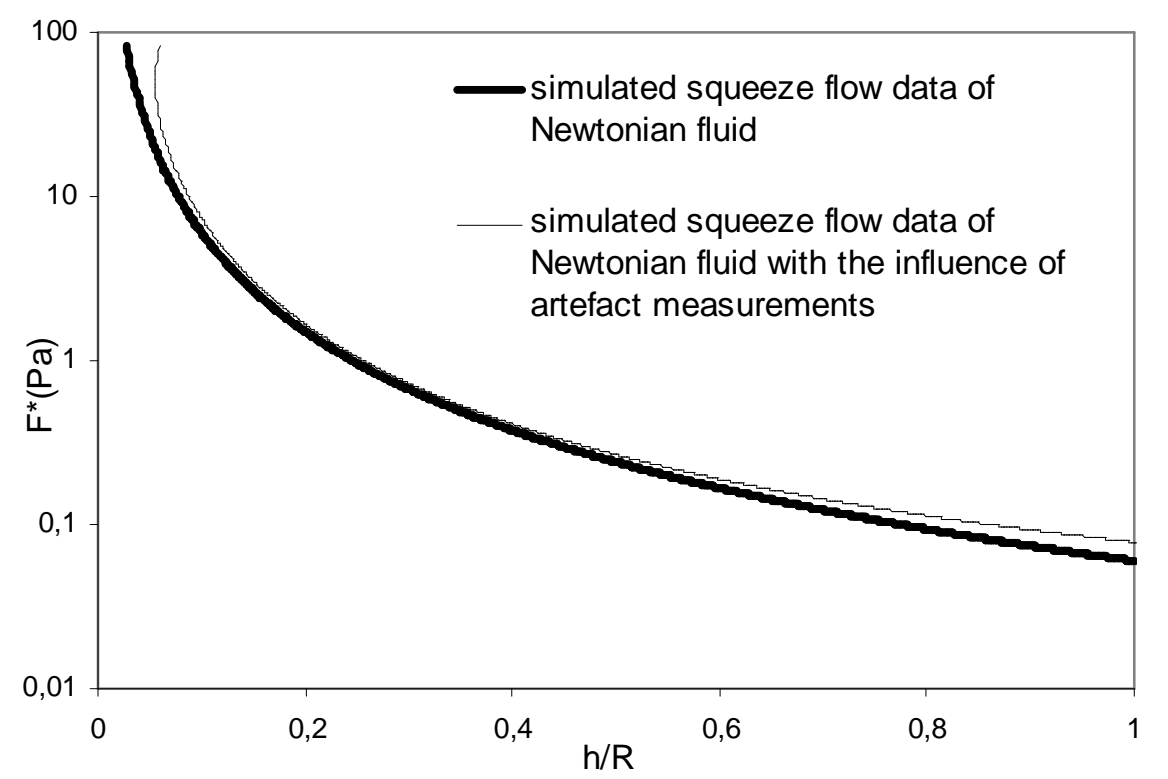

Figure 9. Comparison of the theoretical reduced squeeze force of a Newtonian fluid in simulated sticking flow with (thin line) and without (thick line) the influence of artefact measurements. 


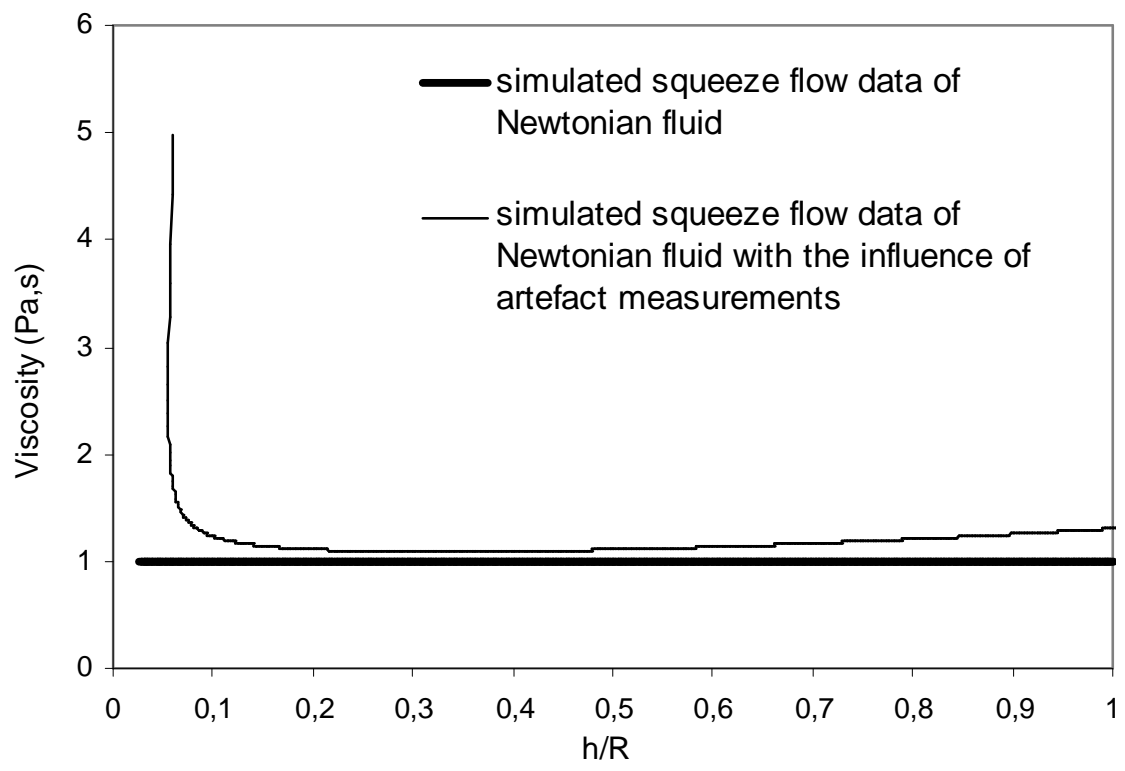

Figure 10. Viscosity of a Newtonian fluid in simulated sticking flow with (thin line) and without (thick line) the influence of artefact measurements. 